\title{
Correction to: Predictors of bowel necrosis in patients with acute mesenteric ischemia: systematic review and meta-analysis
}

\author{
Sameh Hany Emile ${ }^{1}$ [D Sualeh Muslim Khan ${ }^{2} \cdot$ Samer Hani Barsoum ${ }^{1}$
}

Published online: 6 August 2020

(C) Italian Society of Surgery (SIC) 2020

\section{Correction to: Updates in Surgery}

$$
\text { https://doi.org/10.1007/s13304-020-00857-9 }
$$

Table 4: The Column "Biochemical" the entire row "Hyperamylasemia" is missing the updated Table 4 has been copied below

The original article has been updated.

Publisher's Note Springer Nature remains neutral with regard to jurisdictional claims in published maps and institutional affiliations.

The original article can be found online at https://doi.org/10.1007/ s13304-020-00857-9.

Sameh Hany Emile

Sameh200@hotmail.com

Sualeh Muslim Khan

sualeh.muslim@yahoo.com

Samer Hani Barsoum

d.samer.barsoum@gmail.com

1 General Surgery Department, Mansoura University

Hospitals, Mansoura University, PO: 35516, Mansoura,

Egypt

2 Dow Medical College, Dow University of Health Sciences, Karachi, Pakistan 
Table 4 Prognostic score for prediction of bowel necrosis in acute mesenteric ischemia

\begin{tabular}{|c|c|c|c|c|}
\hline \multicolumn{2}{|l|}{ Parameter } & \multirow{2}{*}{$\frac{\text { Odds ratio }(95 \% \mathrm{CI})}{1.11(1.01-1.22)}$} & \multirow{2}{*}{$\frac{\text { Weight (\%) }}{0.8}$} & \multirow{2}{*}{$\frac{\text { Points }}{1}$} \\
\hline Clinical & High body mass index & & & \\
\hline & Pulmonary embolism & $3.0(1.2-7.7)$ & 2.2 & 1 \\
\hline & Coronary heart disease & $3.33(1.17-9.46)$ & 2.4 & 1 \\
\hline & Shock & $3.39(1.09-10.5)$ & 2.4 & 1 \\
\hline & Time between symptoms and operation $>90 \mathrm{~h}$ & $4.8(1.36-9.89)$ & 3.5 & 1 \\
\hline & Organ failure & $8.47(2.75-26.1)$ & 6.1 & 1 \\
\hline & Mesenteric arterial occlusion & $26.5(1.55-453.3)$ & 19.2 & 2 \\
\hline & Systemic inflammatory response syndrome & $28.94(2.29-365.19)$ & 20.9 & 3 \\
\hline \multirow[t]{7}{*}{ Biochemical } & Leukocytosis $>18.1 \times 10(9) / \mathrm{L}$ & $1.22(1.14-1.37)$ & 0.88 & 1 \\
\hline & Hyperamylasemia & $4.22(1.46-12.16)$ & 3.05 & 1 \\
\hline & Serum lactate $>2 \mathrm{mmol} / \mathrm{L}$ & $4.34(1.7-9.2)$ & 3.1 & 1 \\
\hline & Platelet-lymphocyte ratio $>156.26$ & $5.04(1.64-15.49)$ & 3.6 & 1 \\
\hline & Hemoconcentration $\mathrm{Hb}>16 \mathrm{~g}$ & $6(1.58-22.75)$ & 4.3 & 1 \\
\hline & Acidosis with $\mathrm{pH}<7.35$ & $6.4(1.18-58.4)$ & 4.6 & 1 \\
\hline & Neutrophil-lymphocyte ratio $>11.05$ & $7.57(2.47-23.16)$ & 5.5 & 1 \\
\hline \multirow[t]{6}{*}{ Radiological } & Splenic vein thrombosis in CT & $1.6(1.2-2.1)$ & 1.1 & 1 \\
\hline & $\mathrm{SMV}$ thrombosis in $\mathrm{CT}$ & $2.27(1.22-4.19)$ & 1.6 & 1 \\
\hline & Bowel loop dilatation in $\mathrm{CT}$ & $2.29(1-5.25)$ & 1.6 & 1 \\
\hline & Portal vein thrombosis in $\mathrm{CT}$ & $2.7(1.6-4.4)$ & 1.9 & 1 \\
\hline & Free intraperitoneal fluid in $\mathrm{CT}$ & $3.94(2.41-6.46)$ & 2.8 & 1 \\
\hline & Pneumatosis intestinalis in $\mathrm{CT}$ & $10.91(5.24-22.7)$ & 7.9 & 1 \\
\hline
\end{tabular}

$* 0-7=$ Low probability of bowel necrosis; $8-15=$ Moderate probability of bowel necrosis; $16-24=$ High probability of bowel necrosis 